\title{
Macro-micro Interlocked Simulation for Multiscale Phenomena
}

\author{
Kanya Kusano, Shigenobu Hirose, Toru Sugiyama, Shinichiro Shima, \\ Akio Kawano, and Hiroki Hasegawa \\ The Earth Simulator Center, \\ Japan Agency for Marine-Earth Science and Technology \\ 3173-25 Showa-machi, Kanazawa-ku Yokohama, Kanagawa 236-0001, \\ Japan \\ kusano@jamstec.go.jp \\ http://www.es.jamstec.go.jp/esc/research/Holistic/index.en.html
}

\begin{abstract}
A new methodology for the simulation of multiscale processes, called Macro-Micro Interlocked (MMI) Simulation, is introduced. The MMI simulation is carried out by the two-way connection of different numerical models, which may handle macroscopic and microscopic dynamics, respectively. The MMI simulation are applied to several multiscale phenomena, for instance, cloud formation, gas detonation, and plasma dynamics. The results indicate that the MMI simulation provide us an effective and prospective framework for multiscale simulation.
\end{abstract}

Keywords: simulation, macro-micro Interlocked, multiscale, cloud formation, detonation, aurora, plasma, fluid, super-droplet, DSMC.

\section{Introduction}

Multi-scale phenomena, in which the elementary process of micro-scale and the system evolution of macro-scale are tightly connected to each other, quickly comes up as a crucial issue in any state of the art research fields, such as material science, plasma physics, chemistry, astrophysics, geo-science, bio-science and so on. Computer simulation is usually performed on basis of the theoretical description like the partial differential equation, and thus the applicability of simulation model has to be constrained by the limitation of the basic theory behind. So far, the multi-scale simulations have been developed by the two different manners. The first is the extension of macroscopic simulation, in which the microscopic effects are included as phenomenological parameters, just like the anomalous resistivity caused by micro-scale turbulence in high-temperature plasmas and the bulk cloud parameterization in atmospheric global circulation model. However, since the phenomenological parameterization is not guaranteed to work well in unbeknown circumstances, the applicability of such a way would be restricted particularly as an effective tool for prediction of unknown phenomena. 
Another approach is the large-scale microscopic simulation, whereby the macroscopic phenomena are attempted to be built from the elementary block of micro-scale. The large-scale molecular dynamics (MD) simulation is the typical example of that. However, it is computationally so demanding, that the practical application is hardly feasible despite of the incredible capability of the latest super-computers.

Macro-Micro Interlocked (MMI) Simulation is the new type of computation, which is recently introduced by Sato [1]. MMI simulation consists of the macroand micro-simulation models, which are carried out simultaneously and interconnected to each other. The micro-model is performed under the environmental condition controlled by the macro-model, and it feeds back the microscopic information to the macro-model. The fundamental principle of MMI simulation is in common with that of the heterogeneous multiscale method (HMM) 2]. Furthermore, the way to interconnect macro- and micro-scales is similar to Equation-Free approach [3], although, in MMI simulation, we have to adopt some macro-scale model rather than using a coarse projective method. However, originally MMI simulation has been proposed as a computational algorithm suitable to the hardware of heterogeneous architecture, called MMI simulator, in which different types of architecture fitting respectively to the numerical models of macro- and microscales are interconnected. In particular, heterogeneous vector/scalar architecture is the most promising design of the MMI simulator, and we have developed several applications for that.

The objective of this paper is to demonstrate the applications of MMI simulation. For that, we will show the brief results of typical MMI simulations for cumulus cloud formation, gas detonation, and plasma dynamics in the following sections, and the prospects of the MMI simulation will be summarized in Sec.3.

\section{Applications}

\subsection{Cloud Simulation}

Although clouds play a crucial role in meteorological phenomena and climate change like global warming, the numerical modeling of cloud is not well established yet. The reason of that is attributed to the fact that the formation of clouds and the development of precipitation are essentially governed by mutiscalemultiphysics processes. The macro-scale processes such as the fluid motion of moist air associated with clouds is called "cloud dynamics", and the micro-scale processes such as the condensation and coalescence of water droplets are called "cloud microphysics". These two processes mutually affect each other. Numerical methods to simulate the interaction between them should be developed to understand and predict cloud-related phenomena.

Cloud dynamics model to describe the fluid motion of atmosphere has been well developed so far. However, it is still difficult to perform the accurate simulation of cloud microphysics, though several simulation methods, such as bulk parameterization and spectral (bin) methods have been proposed. 
We recently developed a novel simulation model of cloud microphysics, named Super-Droplet Method (SDM) 4], which is a particle-based cloud microphysics model. The super-droplet is defined as computational particle representing multiple real droplets, which have common properties, e.g. position, velocity, cloud condensation nuclear (CCN), and electric charge. The motion of each superdroplet is calculated by the equation of motion or by the assumption that each droplet immediately attains terminal velocity. The condensation and the evaporation of droplets can be directly calculated based on Köhler's theory using the properties of super-droplet and the state variable of atmospheric environment. The coalescence of droplet is handled in stochastic manner.

The cloud dynamics is simultaneously calculated with SDM using the nonhydrostatic model equations,

$$
\begin{aligned}
\rho \frac{D \boldsymbol{U}}{D t} & =-\nabla P-\left(\rho+\rho_{w}\right) \boldsymbol{g}+\lambda \nabla^{2} \boldsymbol{U}, \\
P & =\rho R_{d} T, \\
\frac{D \theta}{D t} & =-\frac{L}{c_{p} \Pi} S_{v}+\kappa \nabla^{2} \theta, \\
\frac{D \rho}{D t} & =-\rho \nabla \cdot \rho, \\
\frac{D q_{v}}{D t} & =S_{v}+\kappa \nabla^{2} q_{v},
\end{aligned}
$$

where $\rho$ is the density of moist air, $\rho_{w}$ the density of liquid water, $\boldsymbol{U}$ the wind velocity, $P$ the pressure, $\lambda$ the viscosity, $R_{d}$ the gas constant for dry air, $T$ the temperature, $\theta$ the potential temperature, $L$ the latent heat, $q_{v}$ the mixing ratio of vapor, $S_{v}$ the source of water vapor, and $\Pi$ is the Exner function, respectively. The density of droplets

$$
\rho_{w}(\boldsymbol{x}, t)=\sum_{i} \xi_{i} m_{i}(t) w\left(\boldsymbol{x}, \boldsymbol{x}_{i}\right),
$$

and the conversion ratio between vapor and liquid

$$
S_{v}(\boldsymbol{x}, t)=-\frac{1}{\rho(\boldsymbol{x}, t)} \frac{\partial \rho_{w}(\boldsymbol{x}, \boldsymbol{t})}{\partial t},
$$

are sent to the fluid model, and used in the source terms, where $i$ is the index of super-droplet, $\xi_{i}$ the multiplicity of super-droplet, $m_{i}$ the mass of droplet, and $w$ is the shape function of super droplet.

SDM is the hybrid model, in which the particle-based direct simulation MonteCarlo (DSMC) model and the fluid model are coupled. By evaluating the integrated squared error for the distribution function in parameter space of droplet properties, we can show that the computational efficiency of SDM is higher than the spectral (bin) method, when more than four properties of cloud droplet are taken into account. (Refer to [4] for further detail.) It implies that the particlecontinuum coupled model is more effective for more complicated system, compared to the conventional methods. 

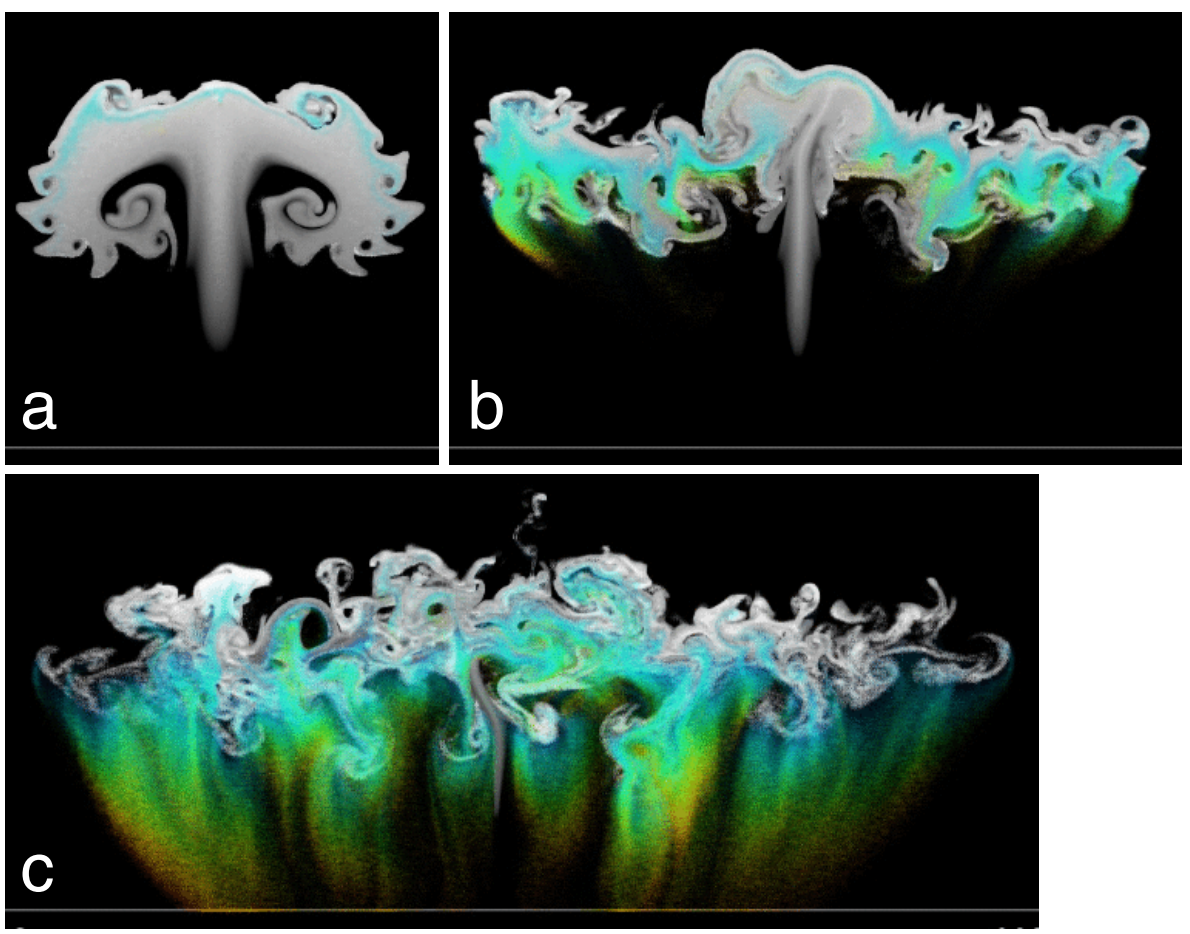

Fig. 1. The distribution of cloud droplets calculated by the coupled simulation of the super-droplet method and non-hydrostatic model. Color represents the size of droplet. (a) At $t=960 \mathrm{sec}$ after simulation starts, upwelling initiated by warm bubble causes the condensation growth of cloud droplets (white particles). (b) At $t=1188 \mathrm{sec}$, droplets bigger than 10 microns in radius (blue particles) further grow due to collisioncoalescence. (c) At $t=1512 \mathrm{sec}$, droplets (yellow particles), which are bigger than 100 micron, are precipitated as rain-drops. Inside the cloud, turbulence-like structure is strongly driven by cumulus convection.

In order to demonstrate the feasibility of SDM, it has been applied to the simulation of cloud formation and precipitation in maritime cumulus. The simulation system is given by 2 -dimensional $x-z$ domain just for simplicity. The initial state consists of un-saturated stratified layer, which is slightly unstable only under $2 \mathrm{~km}$ in altitude, and number of tiny droplets, which contain soluble substance as Cloud Condensation Nuclear (CCN), are uniformly distributed in the entire domain. As shown in Fig 1, the results indicate that the particlecontinuum coupled model may work to simulate the whole process from cloud formation to precipitation without introducing any empirical parameterizations. The new model may provide a powerful tool for the study of cloud-related various problems, although the predictability of SDM should be evaluated more carefully. 


\subsection{Detonation Simulation}

Combustion fluid dynamics is a typical subject of multiscale simulation, in which chemical reaction is mutually interacted with the macroscopic flow dynamics. In the conventional methods, the reaction is treated by the Arrhenius rate equation. However, the reaction rate of the equation must be derived from the distribution function assuming local thermal equilibrium (LTE). Although the assumption could be satisfied in normal condition of fluids, when the local Knudsen number, defined as the ratio of mean free path to characteristic length scale, is larger than 0.01 , the assumption of LTE may not be valid. In the case, the flow should be treated as rarefied one and we have to solve the Boltzmann equation.

Especially, detonation, which is sustained by shock wave driven by combustion wave, is the case, because the thickness of detonation front may be comparable to the mean free path. It implies that the Arrhenius rate equation may not be valid on the detonation front.

So, we have developed a novel method for simulation of combustion by connecting a microscopic molecular model and a macroscopic continuum model, those are based on the Boltzmann equation and the Navier-Stokes equation, respectively. We adopted non-steady DSMC method [5] for the molecular model, and the continuum model is carried out by the HLLC method.

Our detonation model is an extension of Hybrid Continuum-Atomistic Simulation, which has been quickly developed by many authors $[6] 7 / 8$. The algorithm to connect the molecular model and the continuum model is summarized as follows: (1) Gradient of pressure is monitored during the simulation by the continuum model, and the molecular model is embedded in the region where the steepness exceed some threshold and the continuum model is failed. (2) Interlocking layers are laid around the outer-most region of the molecular domains, where particles are generated to let the distribution function be able to reproduce the macroscopic variables, density, velocity and pressure. Numerical flux on the outer-most boundary of the continuum domain is calculated from the particle motion.

Some test results of the two-dimensional detonation simulation are shown in Fig 2 It represents the distribution of pressure on three different snapshots. The detonation front bounded by dotted line corresponds to the domain, where the particle-based model is adopted. The smooth connection between fluid and particle-based models indicates that the continuum-atomistic simulation is applicable also to the combustion process.

\subsection{Plasma Simulation}

Plasma inherently forms a multi-scale system, in which there are different characteristic scales related to electron and ions. The key issue in multiscale plasma processes is how macroscopic magnetohydrodynamics (MHD) is related to microscopic particle kinetics. For instance, in magnetic reconnection that is responsible for explosive energy release in high-temperature plasmas, the kinetics might be important especially in the restricted diffusion region, which is formed when anti-parallel magnetic fluxes collide each other 9 . 


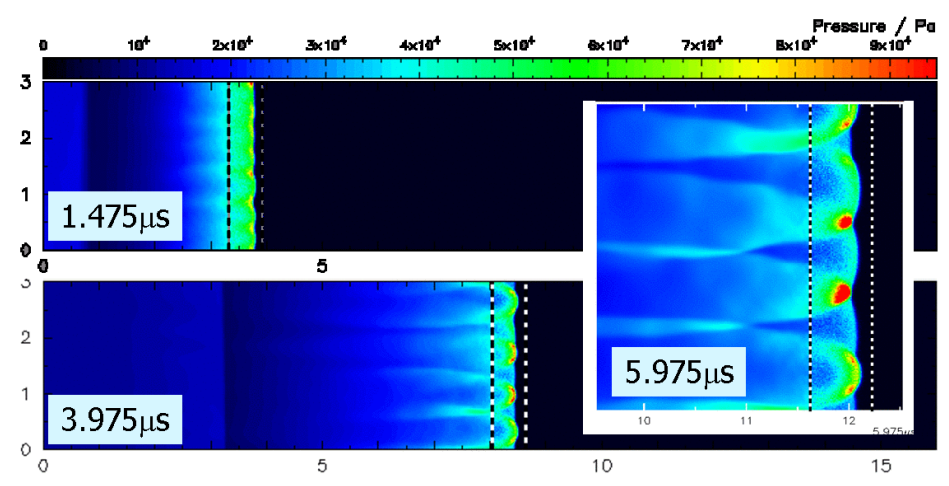

Fig. 2. The pressure distribution of the detonation simulation at three different snapshots. The particle-based domain, which corresponds to the region bounded by dotted lines, tracks the detonation front. The particle-based region at $5.975 \mu \mathrm{s}$ is zoomed-in on right subset.

So, we have developed the new algorithm for such a multiscale plasma dynamics. Our model is constituted by the connection of the particle-in-cell (PIC) model and the magnetohydrodynamic (MHD) model, the former of which is embedded in the MHD simulation domain[10]. This MHD-PIC interlocked model is resembled to the hybrid continuum-atomistic simulation, which was explained in the previous subsection. However, it should be mentioned that, in plasma simulation, several characteristics described by PIC model are negated in continuum (MHD) model, in contrast to the hydrodynamic simulations. So, in order to make the smooth interlocking between MHD and PIC models, we have to introduce some special filter, which passes only the MHD mode from the PIC domain to the MHD domain.

The filtering process is actualized by the following procedure. First, the MHD simulation is advanced from time $t_{n}$ to $t_{n}+\Delta t$ in the whole domain including the PIC domain as the predictor phase (Fig 3 ). Second, the PIC simulation is advanced to $t_{n}+\Delta t$ only in the PIC domain, in which the boundary condition is given by the MHD simulation of the predictor phase. Note that the time step in $\mathrm{PIC}$ is much shorter than that of MHD, and the boundary condition in each PIC step has to be interpolated. The electric field and the electric current density of the PIC result, $\boldsymbol{E}_{P I C}$ and $\boldsymbol{J}_{P I C}$, and those of the MHD predictor results, $\boldsymbol{E}_{M H D}$ and $\boldsymbol{J}_{M H D}$, are mixed as following

$$
\begin{gathered}
\boldsymbol{E}_{*}=w(\boldsymbol{x}) \boldsymbol{E}_{P I C}+(1-w(\boldsymbol{x})) \boldsymbol{E}_{\boldsymbol{M I} \boldsymbol{D}}, \\
\boldsymbol{J}_{*}=w(\boldsymbol{x}) \boldsymbol{J}_{P I C}+(1-w(\boldsymbol{x})) \boldsymbol{J}_{M I \boldsymbol{D}},
\end{gathered}
$$

where $w(\boldsymbol{x})$ is the weighting function that smoothly ramped from 0 to 1 on the periphery of the PIC domain, as shown in Fig 3b. Finally, using $\boldsymbol{E}_{*}$ and $\boldsymbol{J}_{*}$, the MHD simulation is advanced again from $t_{n}$ to $t_{n}+\Delta t$. This is the corrector phase, and the procedure above is repeated until the calculation is finished. In 

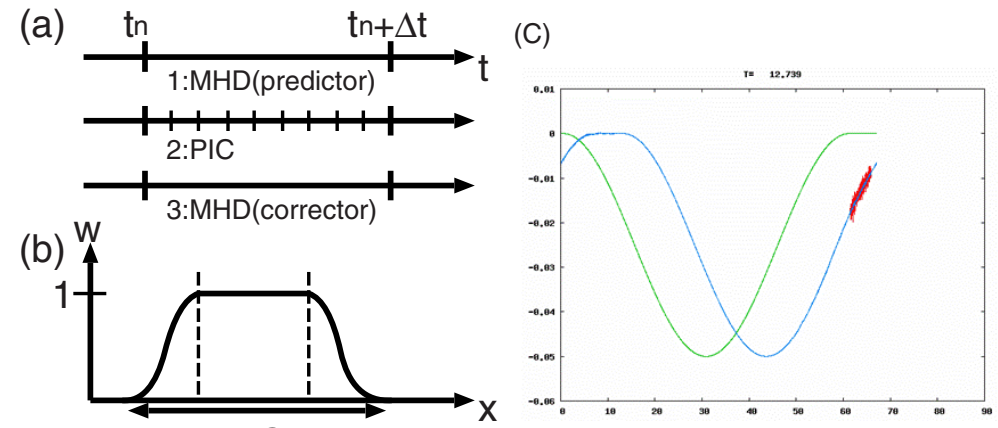

$\mathrm{PIC}$

Fig. 3. (a) The algorithm for time advancement of the MMI plasma simulation. (b) The schematic diagram of spatial relation between the weighting function $w$ and the PIC domain. (c) The result of the test simulation for Alfvén wave propagation.

this algorithm, the kinetic process comes out with $\boldsymbol{E}_{P I C}$ and $\boldsymbol{J}_{P I C}$, which are used instead of the generalized Ohm's law in the corrector phase.

Figure 3r shows the magnetic wave form in the test-simulation for the onedimensional Alfvén wave propagation. Green and blue curves represent the initial state and the result of the MHD model after a while, where red line indicates the result of PIC, which is embedded in a small region. The wave form is scarcely affected by interlocking with the PIC model, as high-frequency modes appear in the PIC domain. It indicates that the particle-continuum connection model is applicable also to plasma simulations.

\section{Summary}

Three different applications for the MMI simulation are demonstrated. In any examples, the particle-continuum connection technique plays a crucial role. However, it is worthwhile to note that the particle-continuum connection in the cloud simulation is not based on the domain decomposition method unlike the other two applications of plasma and detonation, because cloud is ubiquitous phenomena and we cannot restrict the cloud domain into a limited region.

MMI simulation based on the particle-continuum connection technique might be suitable to MMI simulator of heterogeneous architecture. For instance, vector and scalar architectures fit for the algorithms of continuum and particle models, respectively. In fact, the parallelization with MPI is convenient for the implementation of MMI simulation, because the macro and micro models can be assigned to different ranks, and it could be easily ported also to the heterogeneous architecture system.

Although MMI simulator could be a potential tool for multiscale simulation, at least so far the interlocking algorithm has been developed almost in ad hoc manner. Therefore, it is not yet clear what is the best fit architecture for each 
MMI algorithm, and more systematic research for the matching between the algorithm and the architecture of MMI simulator will be required.

Acknowledgments. This work is performed as a part of the program of "Development of Multi-scale Coupled Simulation Algorithm" in the Earth Simulator Center, JAMSTEC.

\section{References}

1. Sato, T.: Macro-Micro Interlocked Simulator, Journal of Physics: Conference Series SciDAC 2005 Scientific Discovery through Advanced Computing, Vol.16, (2005) $310-316$

2. Weinan E, Engquist, B., Li, X., Ren, W., and Vanden-Eijnden,E.: Heterogeneous Multiscale Methods: A Review, Commun. Comput. Phys. 2, 3 (2007) 367-450.

3. Kevrekidis, I.G., Gear, C.W., Hummer, G.: Equation-free: The computer-aided analysis of complex multiscale systems Aiche J. 50, 7 (2004) 1346-1355.

4. Shima, S., Kusano, K, Kawano, A., Sugiyama, T., and Kawahara, S.: Super-Droplet Method for the simulation of Cloud and Precipitation; a Particle-Based Microphysics Model Coupled with Non-hydrostatic Model. submitted to J. Atm. Sci.

5. Bird, G.A.: Molecular Gas Dynamics and the Direct Simulation of Gas Flows. Clarendon Press, Oxford (1994).

6. Aktas, O., Aluru, N.R.: A Combined Continuum/DSMC Technique for Multiscale Analysis of Microfluidic Filters, J. Comp. Phys. 178, 2 (2002) 342-372.

7. Wijesinghe, H.S., Hadjiconstantinou, N.G.: Discussion of Hybrid AtomisticContinuum Methods for Multiscale Hydrodynamics, Int. J. Multiscale Computational Engineering 2, 2 (2004) 189-202.

8. Schwartzentruber, T.E., Boyd, I.D.: A Hybrid Particle-Continuum Method Applied to Shock Waves. J. Comp. Phys. 215, 2 (2006) 402-416.

9. Kusano, K., Maeshiro, T., Yokoyama, T., \& Sakurai, T.: The Trigger Mechanism of Solar Flares in a Coronal Arcade with Reversed Magnetic Shear. Astrophys. j. 610 (2004) 537-549

10. Sugiyama, T., Kusano, K.: Multiscale Plasma Simulation by the Interlocking of Magnetohydrodynamic Model and Particle-in-Cell Kinetic Model. submitted to J. Comp. Phys. 